\title{
ESTUDIO TECNOLOGICO DEL MACERADO DE PERA ( Pyrus communis L.) EN PISCO PROVENIENTE DE LOS VALLES DETACNA
}

\author{
MACERATED TECHNOLOGICAL STUDY OF PEAR (Pyrus communis L.) IN \\ PISCO FROM THE VALLEY OFTACNA
}

\author{
Nicolás Sequeiros Flores'; Rolando Céspedes Rossel ${ }^{2}$
}

\section{RESUMEN}

\begin{abstract}
El presente trabajo de investigación tuvo como fin evaluar el comportamiento tecnológico del macerado de pera (Pyrus communis L.) con piscos provenientes de los valles de Tacna. El pisco inicial tuvo $45^{\circ} \mathrm{GL}$ que con el transcurso del proceso término en 18 $20^{\circ} \mathrm{GL}$; el almíbar se estandarizó a $28^{\circ}$ Brix que por osmosis termino en $24-25^{\circ}$ Brix. La evaluación organoléptica indica que se trata de un producto de buena aceptación en color (4,055), Sabor (3,94), Aroma (4,50) y apariencia general (3,44). El pH se mantuvo constante en 3,45 lo cual contribuyo en la calidad y estabilidad del producto final.
\end{abstract}

Palabras clave: Características organolépticas, Ph, acidez , grado Baume, grado Brix, ${ }^{\circ} \mathrm{GL}$.

\section{ABSTRACT}

\begin{abstract}
The present investigation was aimed at evaluating the technological behavior of macerated pears (Pyrus communis L.) with pisco from the valleys of Tacna. The initial pisco was $45^{\circ} \mathrm{GL}$ with throughout the process finish at $18-20^{\circ} \mathrm{GL}$, I standardize the syrup to $28^{\circ}$ Brix by osmosis finished in 24 to $25^{\circ}$ Brix. The sensory evaluation indicates that it is a product of good color acceptance (4,055), flavor (3.94), Aroma (4.50) and overall appearance $(3,44)$. The $\mathrm{pH}$ was kept constant at 3.45 which contributed to the quality and stability of the final product.
\end{abstract}

Keywords: Characteristics organoleptic, $\mathrm{pH}$, acidity, degree Baume, Brix, ${ }^{\circ} \mathrm{GL}$

\section{I.- INTRODUCCIÓN}

En los tiempos actuales cada vez es más evidente que el Perú no es ajeno a la globalización y a los cambios que ésta conlleva. Se vuelve trascendental participar competitivamente en el mercado internacional, aprovechando la fuerte tendencia a la liberalización de aranceles en pro del fortalecimiento de los intercambios comerciales. En este contexto, motivados por la fuerte atención que el Pisco ha causado en el ambiente peruano, hemos visto por conveniente focalizar nuestra investigación en el desempeño del Pisco en el macerado de pera. Así el trabajo tiene como objetivo general elaborar un macerado de pera utilizando el pisco Tacneño. Entre las tendencias de mercado se encontró la demanda creciente por sabores inusuales, que cobra popularidad en las bebidas para coctelería, y el deseo de experimentar con diferentes clases de bebidas por parte de los jóvenes. Ello, abre una ventana de oportunidad para el ingreso de nuevos productos que puedan acomodarse a esta tendencia de gustos. Debido a lo anterior se ha establecido posicionar al pisco y macerado como un licor dirigido a la coctelería, para lo cual se introduciría una línea de macerados de pera constituida por las variedades producidas en la zona. El mercado de licores presenta una compleja estructura normativa, estatal y local en las regulaciones y requerimientos legales y tributarios, por lo que el conocerlos se convierte en un factor decisivo de éxito.

Por ello, es necesario focalizar la estrategia de ingreso a un mercado o a una limitada cantidad de mercados, y evaluar respecto a qué normativas son más recomendables y/o favorables, antes de realizar esfuerzos de penetración del producto en el mercado. Uno de los métodos tradicionales de la licorería, son los macerados puesto que se dan diferentes formas de preparar estos licores. P

El procesamiento de los piscos y macerados es un arte tradicional. Este tipo de licores se puede 
encontrar en mercados, supermercados y licorerías de la ciudad. Conviene advertir que en el área de influencia del proyecto no existe actualmente ninguna empresa o planta industrial que procese y oferte al mercado el producto que pretende producir el trabajo de investigación. Los Piscos y Macerados, más bien existen en volúmenes de producción a pequeña escala, elaborado en forma artesanal o casera, que no reúne la calidad que desearía el público consumidor, cual es: materia prima seleccionada, higiene y calidad en su pureza; pero se ha podido detectar que gran parte de la producción de este tipo de licor es elaborado bajo condiciones que además antihigiénicas, son mezclados con otros ingredientes (agua, azúcar, etc.), por lo tanto, adulterados en gran proporción, el mismo que ha sido determinado por observación en los diferentes centros de abasto de la ciudad de Tacna y otras ciudades como Arequipa, Moquegua e Ilo.

\section{OBJETIVO}

El objetivo de la presente investigación fue obtener un macerado de pera de buena calidad y determinar los estándares de elaboración según las buenas prácticas de procesamiento.

\section{II.- MATERIALES Y MÉTODOS}

\subsection{Descripción}

El presente estudio es de tipo descriptivo y experimental, considerando como unidad de estudio la pera proveniente de los fundos productores de Tacna, habiéndose acopiado la misma de los Mercados de abasto. Las muestras seleccionadas fueron procesadas en los Laboratorios de la escuela académica de la Facultad de Ciencias Agropecuarias - UNJBG.

\subsection{Materiales}

Materia prima e insumos: peras de adecuada madurez Insumos: ácido cítrico, azúcar, pisco puro $45^{\circ}$, agua, estabilizador. Equipos e instrumentos de medición: Refractómetro ABBE escala o $30^{\circ} \mathrm{Brix} \mathrm{Ph}-$ metro digital, Balanza, Termómetro escala de $\mathrm{O}-100^{\circ} \mathrm{C}$, cocina industrial, utensilios diversos(ollas, cuchillo, posillos, coladores, otros), frascos de vidrio con tapa rosca Capacidad $1 \mathrm{~L}$.

\subsection{Metodología}

Los procedimientos de elaboración del macerado se describen a continuación y se muestra en el Gráfico $\mathrm{N}^{\circ} \mathrm{O} 1$.

\section{- Recepción}

Para la elaboración del macerado de pera tenemos dos insumos importantes, el destilado Pisco y la fruta. El destilado se obtiene de la uva y la pera es recepcionada en canastillas provenientes de las zonas productoras de Tacna.

\section{- Selección}

La selección consiste en separar todas aquellas frutas que se encuentren demasiado maduras o inmaduras, separar toda fruta que presente algún tipo de daño. Se prefiere para esto frutas sanas, carnosas, no muy maduras se consideran las de mejor consistencia porque son estas las que soportan mejor el calor y la maceración.

\section{- Limpieza}

Una vez seleccionadas las frutas, se las limpia con un trapo para quitarles el polvo, y si fuera necesario se procede a darles un pre-lavado y desinfección con agua clorada.

\section{- Macerado}

La finalidad de extraer los aromas y otras sustancias solubles de menor importancia, para los fines que se persiguen. Esto se lleva a cabo mediante los fenómenos de osmosis y difusión. Se junta el destilado con la fruta y se deja macerar por el tiempo que se considere necesario aproximadamente seis semanas a temperatura ambiente y herméticamente cerrados.

\section{- Edulcorado}

En este proceso se procede a agregar el jarabe hasta lograr el estandarizado que aproximadamente debe de estar entre 22 a 2.5 ${ }^{\mathrm{o}}$ Brix.

\section{- Filtrado}

Una vez realizada la edulcoración, se procede a filtrar utilizando como filtrante papel filtro y bomba de vacío. Con esto se va a dar un clarificado y abrillantado al producto.

\section{- Ajuste}

En este proceso se ajusta el grado alcohólico y las demás características sensoriales.

\section{- Envasado y etiquetado}

Para el envasado se utiliza envases de vidrio 
cilíndricos de capacidad de $380 \mathrm{ml}$ que son llenados con fruta y cubiertos con el licor. Una vez llenados son cerrados herméticamente y etiquetado.

\section{- Almacenaje}

A temperatura ambiente.

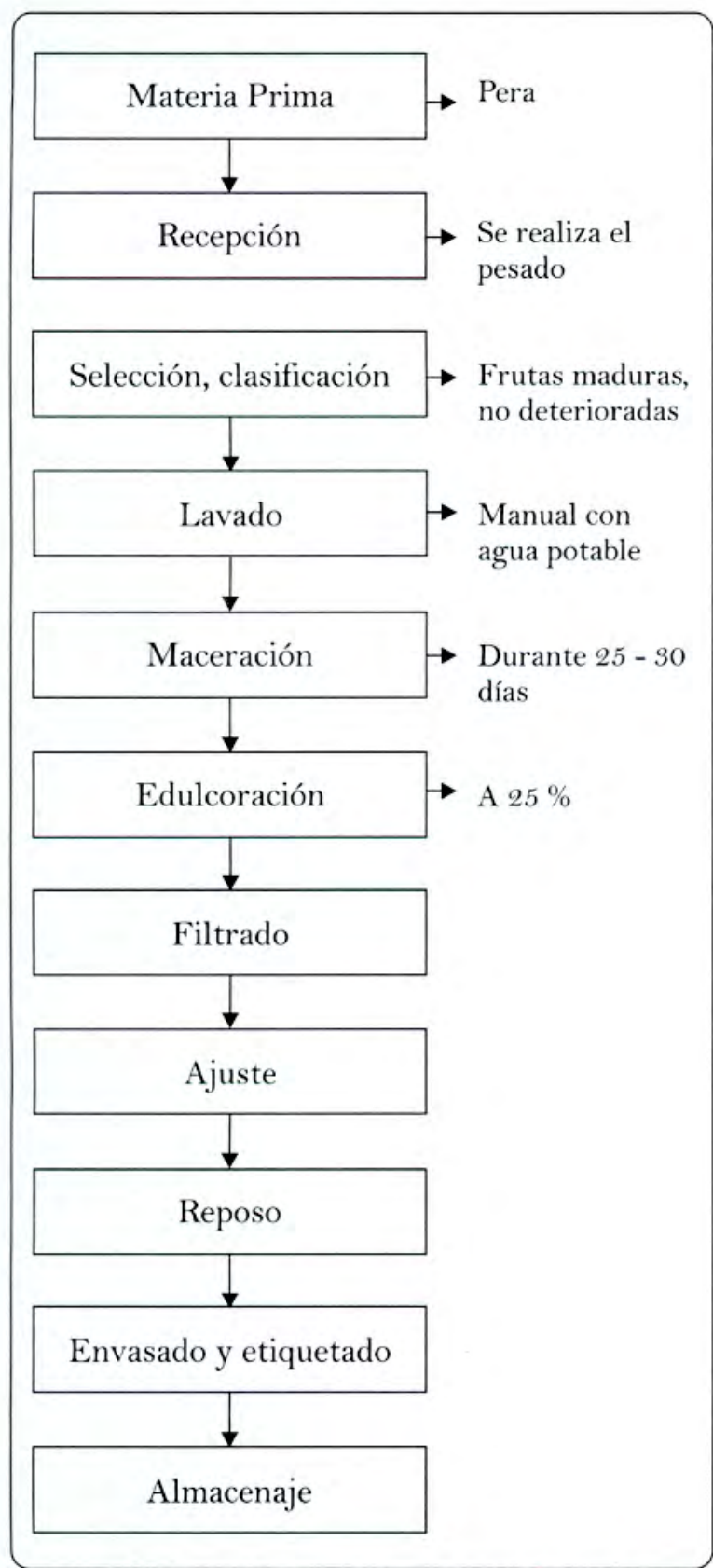

Gráfico $\mathbf{N}^{0}$ 01. Elaboración de macerado de pera.

\section{III.- RESULTADOS}

El producto proveniente de chacra no es uniforme, teniendo como una población de dos jabas de $25 \mathrm{Kg}$ se tiene la Tabla $\mathrm{N}^{\circ} \mathrm{O} 1$ :
Tabla $\mathbf{N}^{\mathbf{o}}$ 01. Rendimiento de pera ( $25 \mathrm{Kg}$ de pera / jaba)

\begin{tabular}{|c|c|}
\hline Calidad & Rendimiento de pera / jaba \\
\hline A & 19 \\
\hline B & 16 \\
\hline C & 15 \\
\hline
\end{tabular}

Fuente: Elaboración propia

\section{Donde:}

A: Peras de optima madurez y tamaño regular.

B: Peras surtidas en tamaño y madurez.

C: Peras de tamaño pequeño y sobre maduras.

Caracterización de la materia prima:

En la Tabla $\mathrm{N}^{\circ} \mathrm{O} 2$ se muestra la relación entre el grado de madurez, la concentración de azúcar y el Ph de la pera.

Tabla $\mathbf{N}^{\circ} \mathbf{0 2}$. Relación entre la madurez, los grados Brix y $\mathrm{Ph}$ de la pera.

\begin{tabular}{|l|c|c|}
\hline \multicolumn{1}{|c|}{ Grado de madurez } & $\begin{array}{c}\text { Concentración de } \\
\text { azúcar }{ }^{\circ} \text { Brix }\end{array}$ & $\mathrm{Ph}$ \\
\hline Verdes & 8 & 3,5 \\
\hline Estado óptimo de madurez & 12 & 3,7 \\
\hline Sobre maduras & 11 & 4,0 \\
\hline
\end{tabular}

Fuente: Elaboración propia

En la Tabla $\mathrm{N}^{\circ} 03$ se muestra las cantidades de azúcar que es necesario adicionar a la pera para alcanzar los niveles de $24-26^{\circ}$ Brix del almíbar.

Tabla $\mathbf{N}^{\circ}$ os. Grados ${ }^{\circ}$ Brix de la pera $\mathrm{y}$ azúcar a ser agregado.

\begin{tabular}{|c|c|}
\hline $\begin{array}{c}\text { Grados } \\
{ }^{\circ} \text { Brix }\end{array}$ & $\begin{array}{c}\text { Kg de azúcar a agregar por litro } \\
\text { para llegar a } 24 \text { y } 26^{\circ} \mathrm{Brix}\end{array}$ \\
\hline 10 & $0,168-0,192$ \\
\hline 10,5 & $0,160-0,184$ \\
\hline 11 & $0,152-0,176$ \\
\hline 11,5 & $0,147-0,171$ \\
\hline 12 & $0,142-0,166$ \\
\hline 12,5 & $0,0,139-0,163$ \\
\hline 13 & $0,131-0,155$ \\
\hline 13,5 & $0,126-0,150$ \\
\hline
\end{tabular}

Fuente: Elaboración propia

Ciertas operaciones unitarias más importantes son: 
- Maceración: Esta operación consistió en la extracción de aromas u otras sustancias volátiles de la fruta que se transfieren al pisco para obtener un licor aromático de sabor característico a la fruta, esto se realiza mediante el proceso osmótico entre la fruta y el pisco. Se hace una mezcla del pisco $\left(45^{\circ}\right)$ y la fruta escaldada dejando macerar por 25 - 30 días a temperatura ambiente y en recipientes de vidrio herméticos. La fruta empleada fue entre 30 $50 \%$ del volumen del contenido.

- Estandarización: Consistió en regular el grado alcohólico $\left({ }^{\circ} \mathrm{GL}\right)$ y la concentración de azúcar ( $\left.{ }^{\circ} \mathrm{Brix}\right)$. El porcentaje de alcohol del producto final es entre $16-20^{\circ} \mathrm{GL}$. La concentración de azúcar queda regulada con la adición de almíbar esta entre $25-30^{\circ}$ Brix.

La estandarización consistió en las siguientes etapas:

- El almíbar es el resultado de mezclar el azúcar con el agua, el agua debe estar hervida previamente. La cantidad de azúcar empleada fue entre $0,6-0,8 \mathrm{Kg}$ por litro de agua para lograr $28-30^{\circ}$ Brix.El almíbar se enfría y se cuela para eliminar impurezas.

- Adición de ácido cítrico.- La finalidad fue resaltar el sabor y el aroma de la fruta, se regula el $\mathrm{Ph}$ a 3,5 se adiciona entre $1-3 \mathrm{~g}$ por litro de macerado.

- Formulación de la mezcla pisco: Almíbar, se ha empleado una cantidad de almíbar entre 37 $42 \%$ del volumen total de macerado.

Las proporciones de fruta/almíbar/ pisco se realizó por triplicado con la posibilidad de encontrar alguna variación en el gusto o alguna característica organoléptica del producto final. Los resultados se muestran en la Tabla $\mathrm{N}^{\circ} 04$ y en la Tabla $\mathrm{N}^{\circ} 05$ respectivamente.

Tabla $\mathbf{N}^{\circ}$ 04. Proporciones de fruta, almíbar $y$ pisco en tres tratamientos de macerado de Pera $(\mathrm{g} / \mathrm{ml})$.

\begin{tabular}{|c|c|c|c|}
\hline Tratamiento & fruta & Almíbar & Pisco \\
\hline \multirow{3}{*}{1} & 350 & 325 & 325 \\
& 350 & 390 & 260 \\
& 350 & 260 & 390 \\
\hline \multirow{3}{*}{2} & 400 & 260 & 390 \\
& 400 & 325 & 325 \\
& 400 & 195 & 455 \\
\hline \multirow{3}{*}{3} & 450 & 290 & 350 \\
& 450 & 275 & 375 \\
& 450 & 195 & 455 \\
\hline
\end{tabular}

Fuente : Elaboración propia
Tabla $\mathbf{N}^{\mathbf{0}} \mathbf{0 5}$. Valores de Grados Brix, grado alcohólico y $\mathrm{pH}$ del macerado de pera

\begin{tabular}{|c|c|c|c|}
\hline Tratamiento & $\begin{array}{c}\text { Grados } \\
\text { Brix }\end{array}$ & ${ }^{\circ} \mathrm{GL}$ & $\mathrm{pH}$ \\
\hline \multirow{3}{*}{1} & 23,5 & 17 & 3,5 \\
& 24,8 & 19 & 3,0 \\
& 23,5 & 21 & 3,6 \\
\hline \multirow{2}{*}{2} & 25,5 & 18 & 2,8 \\
& 24,0 & 15 & 3,0 \\
& 20,5 & 18 & 3,5 \\
\hline \multirow{3}{*}{3} & 27,5 & 20 & 3,7 \\
& 24,0 & 17 & 3,5 \\
& 26,5 & 18 & 3,8 \\
\hline
\end{tabular}

Fuente: Elaboración propia

La tabla $\mathrm{N}^{\circ} \mathrm{O6}$ nos muestra los resultados de la evaluación organoléptica.

Tabla $\mathbf{N}^{\mathbf{0}}$ 06. Análisis Organoléptico del Macerado de Pera

\begin{tabular}{|c|c|c|c|c|}
\hline Tratamiento & Color & Sabor & Aroma & $\begin{array}{c}\text { Apariencia } \\
\text { General }\end{array}$ \\
\hline \multirow{3}{*}{1} & 4,0 & 5,0 & 4,5 & 3,5 \\
& 3,0 & 3,5 & 5,0 & 4,0 \\
& 4,5 & 4,0 & 5,0 & 3,0 \\
\hline \multirow{3}{*}{2} & 4,5 & 4,0 & 4,0 & 3,5 \\
& 3,5 & 3,0 & 3,5 & 3,0 \\
& 4,0 & 4,0 & 4,0 & 3,0 \\
\hline \multirow{3}{*}{3} & 3,5 & 4,5 & 5,0 & 4,0 \\
& 5,0 & 4,0 & 4,5 & 3,5 \\
& 4,5 & 3,5 & 5,0 & 3,5 \\
\hline
\end{tabular}

Fuente: Elaboración propia

\section{IV.-DISCUSIÓN}

Según la Tabla $\mathrm{N}^{\circ} \mathrm{O} 2$ las características organolépticas del fruto esta en función de la madurez.

De los resultados podemos indicar que existe una evolución favorable en las características organolépticas del producto, siendo la más destacada el aroma, esto significa que hubo un buen proceso de osmosis.

Por otro lado, los jueces detectaron una falta de presentación o apariencia general, lo cual se demuestra en la calificación baja, esto a su vez nos demuestra que debió utilizarse un clarificante, también fue objetado la presentación del producto en trozos con cáscara ; la eliminación de la misma pudo atentar contra la integridad de la pulpa que podría desprenderse en desmedro de la calidad .

La elaboración de macerados en otros productos es similar, más se desconoce los resultados de evaluación en este aspecto y las 
presentaciones también son variables dado que la pera no tiene la suficiente cantidad de azúcar o bien son demasiado ácidas según la madurez, para obtener un líquido agradable se le tiene que agregar azúcar a niveles de $24-26{ }^{\circ}$ Brix, sin embargo a niveles de azúcar de la fruta no habrá necesidad de adicionar azúcar, esto se muestra en la Tabla $\mathrm{N}^{\circ} 03$.

El escaldado consistió en sumergir la pera en agua a temperatura de ebullición por $5-7 \mathrm{~min}$ dependiendo de la madurez. La finalidad fue eliminar carga microbiana, inactivar enzimas, estabilizar el color y producir mayor permeabilidad de la piel para mejorar y acelerar la maceración. Esta operación consiste en la extracción de aromas $\mathrm{u}$ otras sustancias volátiles de la fruta que se transfieren al pisco y obtener un licor aromático de sabor característico a la fruta, el miso que se realiza mediante el intercambio de componentes de la fruta y el pisco. Se hace una mezcla de pisco $\left(45^{\circ}\right)$ y la fruta escaldada se debe macerar por 25 - 30 días a temperatura ambiente y en recipientes de vidrio. La fruta empleada fue entre $30-50 \%$ del volumen del contenido.

La estandarización consistió en regular el grado alcohólico ( ${ }^{\circ} \mathrm{GL}$ ) y la concentración de azúcar ( ${ }^{\circ}$ Brix).

El porcentaje de alcohol del producto final quedó entre $16-20^{\circ} \mathrm{GL}$.

La concentración de azúcar se reguló con la adición de almíbar esta entre $25-30^{\circ} \mathrm{Brix} \mathrm{La}$ estandarización consistió en las etapas:

- El almíbar es el resultado de mezclar el azúcar con el agua, el agua debe estar hervida previamente. La cantidad de azúcar empleada fue entre $0,6-0,8 \mathrm{Kg}$ por Litro de agua para lograr concentraciones de $28-30^{\circ}$ Brix. se enfría y se cuela para eliminar impurezas.

Adición de ácido cítrico, la finalidad fue resaltar el sabor y el aroma de la fruta, se reguló el $\mathrm{Ph}$ a 3,5 mediante el ácido. Según las Tablas 4 y 5 que son necesarios manejar las proporciones de fruta, pisco y almíbar.

Los resultados finales encontrados nos indicaron que estos corresponden a los parámetros de un producto de este tipo, es decir, se encuentran dentro de los márgenes permitidos y conocidos para este producto.

La influencia del tenor ácido del producto mostrado en la Tabla $\mathrm{N}^{\circ} 05$ contribuye favorablemente en el sabor y gusto del producto final, no habiendo reportes de estos estudios se sugiere se tengan en cuenta los encontrados en el presente estudio.

Según la Tabla $\mathrm{N}^{\circ}$ 06, el aroma característico de la pera es la detectada por los jueces.

\section{V.-CONCLUSIONES}

- Se concluye que la hipótesis planteada queda demostrada pues es posible obtener un producto como el macerado de pera de buena aceptación.

- El escaldado a $100^{\circ} \mathrm{C}$ por tiempos menores a 35 min no permiten lograr el objetivo, sin embargo tiempos de $5-7 \mathrm{~min}$ sí permiten obtener una buena fijación del color y ablandamiento de la pulpa para una osmosis más eficaz.

- Se concluye también que los frutos de cierta dureza como la pera precisan de mayores tiempos de tratamiento térmico.

- Definitivamente la incorporación y regulación de la acidez ( $3 \mathrm{~g} / \mathrm{L}$ ) permiten mejorar la calidad organoléptica y a la vez permiten una buena estabilidad del producto final.

- Contenidos mayores de grado alcohólico ( 40 $45^{\circ} \mathrm{GL}$ ) en el producto desmerecen su calidad, dado que resalta el sabor alcohólico cuando este producto debe ser matizado por el sabor dulce.

- El almíbar debe prepararse en los rangos de 28 $30{ }^{\circ}$ Brix siendo este relativamente alto, sin embargo, durante el macerado se produce la transferencia de éste hacia la pulpa conjuntamente con el pisco lográndose una pulpa con sabor entre alcohólico y dulce.

- Se concluye también que la presencia de cáscara en la pulpa desmerece la calidad y apariencia general del producto final.

\section{REFERENCIAS BIBLIOGRÁFICAS}

[1] Arata A. y Torres O. ( 2008) Rumbo a la competitividad, promoción de la Agroindustria Lima DESCO. Alternativas de desarrollo.

[2] Escobar E. Jaime ( 2009 ) Elaboración de vinos . Editorial Acribia S.A. Zaragoza España.

[3] Huamán G. (2002) Destilado de la pera . 
alternativa de desarrollo agroindustrial . Serie Promoción y Desarrollo. Lima - Perú.

[4] Kolb Erick (2005) Elaboración de vinos. Editorial Acribia Zaragoza España.

[5] Norma Técnica Peruana NTP (2002) Bebidas alcohólicas. Pisco . Requisitos $6^{\circ}$ Edición Lima-Perú.

[6] Puerta Alex ( 2003 ) Elaboración del vino. Proyecto Sam Martin Lima-Perú.

[7] Reglamento de Control sanitario de bebidas (2005) D.S. 07-05 Lima Peru.
[8] Rodríguez C. Marco ( 2004) Efecto de la variedad y madurez de la uva en la fermentación sobre la presencia de esteres del pisco. Universidad de San Agustín Arequipa-Perú.

[9] Soto P. Rogelio ( 2005 ) Como crear tu empresa de vinos y licores. Editorial Palomino E.I.R.L. Lima Perú.

\section{Correspondencia:}

Nicolás Sequeiros Flores

Ciudad Universitaria fundo "Los Granados" Av. Miraflores s/n Tacna - Perú

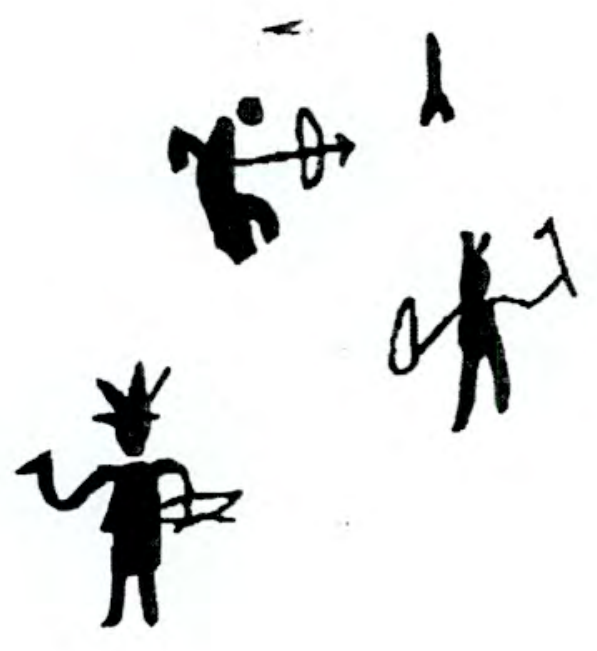

